\title{
Impact of Chylous Ascites on Colon Cancer in Laparoscopic Surgery
}

\author{
Shinya Munakata*, Kazuhiro Sakamoto, Kumpei Honjo, Masaya Kawai, Kiichi Sugimoto, \\ Masakazu Ouchi, Makoto Takahashi, Yutaka Kojima, Kunihiko Nagakari, Yuichi Tomiki \\ Department of Coloproctological Surgery, Juntendo University Faculty of Medicine, Tokyo, Japan \\ Email: *smunaka@juntendo.ac.jp
}

How to cite this paper: Munakata, S., Sakamotom, K., Honjo, K., Kawai, M., Sugimoto, K., Ouchi, M., Takahashi, M., Kojima, Y., Nagakari, K. and Tomiki, Y. (2018) Impact of Chylous Ascites on Colon Cancer in Laparoscopic Surgery. Open Journal of Gastroenterology, 8, 269-274. https://doi.org/10.4236/ojgas.2018.87029

Received: June 19, 2018

Accepted: July 24, 2018

Published: July 27, 2018

Copyright $\odot 2018$ by authors and Scientific Research Publishing Inc. This work is licensed under the Creative Commons Attribution International License (CC BY 4.0).

http://creativecommons.org/licenses/by/4.0/

\begin{abstract}
Objectives: To investigate the epidemiology and risk factors of chylous ascites. Methods: We identified the cases of 913 consecutive patients who under went urgery for colorectal cancer at our University Hospital between January 2005 and December 2016. We divided the patients into those with and those without chylous ascites and compared the two groups by age, gender, body mass index, tumor location, $\mathrm{T}, \mathrm{N}$ factor, stage, operation time, intra-operative bleeding, and duration of postoperative hospital stay. Results: Chylous ascites developed in 8 of the 913 patients $(0.8 \%)$. Neither age, sex, tumor location, and body mass index nor number of lymph nodes were associated with postoperative chylous ascites. Ascites occurred significantly less frequently in the early stage of colorectal cancer than in the late stage $(p=0.04)$. There was no significant difference between the groups in operative factors, including operation time and blood loss. Postoperative hospital stays were longer in patients with chylous ascites (20.5 days) than in those without (11 days) $(\mathrm{p}=0.02)$. Conclusions: Late stage was one of the risk factors for chylous ascites in the present study. Other risks, such as tumor location and blood loss, will vary from institution to institution.
\end{abstract}

\section{Keywords}

Chylous Ascites, Colon Cancer, Epidemiology, Laparoscopic Surgery, Risk Factor

\section{Introduction}

The number of colorectal cancer (CRC) patients has been increasing rapidly worldwide in recent decades. Curative resection is important, and major complications are well reported, including surgical site infection, pneumonia, pul- 
monary embolism, renal insufficiency, cerebrovascular accident, sepsis, anastomotic leakage, and ileus [1]. However, postoperative chylous ascites after colorectal cancer surgery is a rare problem, with an incidence ranging from $1.0 \%$ to $6.6 \%$ of patients who have undergone such surgery [2] [3]. Chylous ascites is defined as the extravasation of milky or creamy peritoneal fluid rich in triglycerides [4].

Most authors consider a patient to have chylous ascites if the fluid contains a minimum of $150 \mathrm{mg} / \mathrm{dl}$ or $200 \mathrm{mg} / \mathrm{dl}$ in triglycerides [5]. Others use laboratory findings of a drainage fluid/serum ratio greater than 1.0 for triglycerides [6]. Postoperative chylous ascites is caused by direct surgical trauma to the major lymphatic tributaries. Recent studies showed that long-term outcomes were not affected by the presence of chylous ascites [7]. However, chylous ascites is known to prolong hospital stays by necessitating treatment with total parenteral nutrition or a medium-chain triglyceride diet [5]. Little is known about the risk factors or optimal management strategy for chylous ascites. Some studies reported that right hemicolectomy or tumors fed by the superior mesenteric artery were associated with the development of chylous ascites after colorectal cancer surgery [2] [8]. Another study showed that it correlates with the number of lymph nodes harvested, concomitant vascular resectionor manipulation of the para-aortic area, retroperitoneal tumor invasion, and increased blood loss after major abdominal surgery [5].

In the present study, we analyzed our experience with chylous ascites and proposed its epidemiology, risk factors, and beneficial treatment approaches.

\section{Patients and Methods}

Patients with clinically confirmed stage I - IV colon cancer diagnosed at ourhospital between January 2005 and December 2016 were eligible to be in this study. This patient series included those who underwent laparoscopic primary tumor resection. In stage IV patients, it did not matter whether simultaneous curative resection of metastases occurred or not. Patients who had double cancer, non-elective surgery, pre-operative radiotherapy, or chemotherapy, or who showed clinical evidence of infection or other inflammatory conditions, were excluded from the study.

After surgical resection, all specimens were his to pathologically reviewed, and the pathological classification and stage were determined according to the classification established by the American Joint Committee on Cancer (AJCC) TNM staging system.

We divided the patients into two groups: Group A, having chylous ascites ( $\mathrm{n}=$ 8), and Group B, nonchylous ascites $(\mathrm{n}=905)$. We compared the groups according to these factors: age, gender, body mass index, tumor location, $\mathrm{T}, \mathrm{N}$ factor, stage, operation time, intra-operative bleeding, and duration of postoperative hospital stay. This retrospective study was approved by our hospital's Institutional Review Board, and the requirement for patient consent was waived. 


\section{Statistical Analyses}

The categorical variables were compared using the chi-square test or Fisher's exact test as appropriate. Continuous variables are presented as median values and were compared using the Mann-Whitney U-test. Data were analyzed using JMP 10 software (SAS, Cary, NC, USA).

\section{Results}

We defined chylous ascites as containing a minimum of $150 \mathrm{mg} / \mathrm{dl}$ in triglycerides. Chylous ascites developed in 8 of the 913 patients $(0.8 \%)$ who underwent laparoscopic colon cancer surgery. The study group was 623 males and $390 \mathrm{fe}-$ males aged 28 - 90 years (median 65 years). The tumors were located in the cecum (C) in 77 patients (8.4\%), followed by the ascending colon (A) in 171 patients $(18.7 \%)$, transverse colon $(\mathrm{T})$ in 89 patients $(9.7 \%)$, descending colon (D)in 56 patients $(6.1 \%)$, sigmoid colon (S) in 348 patients $(38.1 \%)$, and recto-sigmoid (RS) in 172 patients (18.8\%). The TNM 7 th T categories were T1 in 33 patients (3.6\%), T2 in 429 patients (47.0\%), T3 in 415 patients (45.5\%), and $\mathrm{T} 4$ in 36 patients (3.9\%).

Regarding TNM staging, 40 patients (4.4\%) were stage I, 418 patients (46.1\%) were stage II, 396 patients (43.7\%) were stage III, and 51 patients $(5.6 \%)$ were stage IV.

Table 1 showed the clinical characteristics of the 8 patients who hadchylous ascites. Representative chylous ascites is shown in Figure 1. In all of those patients, ascites was discovered after they had already started a normal diet in postoperative day $4-8$. All patients were switched to fasting then switched to a low-fat diet. No pat0ients were treated with a continuous intravenous drip after the development of chylous ascites. For summary see Table 2. The patient and tumor characteristics were compared among the 8 patients with chylous ascites and the 905 patients without (Table 2). Neither age, sex, tumor location, body mass index, nor number of lymph nodes were associated with postoperative

Table 1. Characteristics and outcomes of the patients with chylous ascites (C: cecum, A: ascending, T: transverse, D: descending, S: sigmoid).

\begin{tabular}{cccccccccc}
\hline Age & Sex & Location & Stage & Blood loss & Operation time & BMI & Date of onset & No of lympho node & Postoperative day \\
\hline 50 & F & S & II & 10 & 286 & 26.1 & 7 & 22 & 12 \\
70 & F & A & III & 60 & 450 & 19.6 & 8 & 23 & 22 \\
64 & F & D & III & 40 & 350 & 19.8 & 8 & & 27 \\
63 & M & S & III & 10 & 166 & 22.4 & 4 & 15 & 12 \\
52 & M & C & III & 25 & 238 & 26.3 & 4 & 19 & 19 \\
50 & M & T & III & 55 & 269 & 21.3 & 4 & 34 & 19 \\
53 & F & S & III & 13 & 290 & 18.6 & 7 & 50 \\
72 & M & A & IV & 32 & 255 & 22.0 & 6 & 16 & 18 \\
\hline
\end{tabular}


Table 2. Univariate analyses of characteristics and outcomes of the patients with and without chylous ascites (C: cecum, A: ascending, T: transverse, D: descending, S: sigmoid).

\begin{tabular}{cccc}
\hline & Chylous $(\mathrm{n}=8)$ & non-Chylous $(\mathrm{n}=905)$ & p value \\
\hline Age & 58 & 66 & 0.1 \\
Sex (M/F) & $4 / 4$ & $519 / 386$ & 0.67 \\
BMI & 21.6 & 22.9 & 0.32 \\
Location (C/A/T/D/S/RS) & $1 / 2 / 1 / 1 / 3 / 0$ & $76 / 169 / 88 / 55 / 345 / 172$ & 0.86 \\
Stage & $0 / 1 / 6 / 1$ & $40 / 418 / 396 / 51$ & 0.04 \\
Operation time (min) & 277.5 & 246 & 0.16 \\
Blood loss (g) & 28.5 & 25 & 0.79 \\
No. of lymph node & 20.5 & 18 & 0.09 \\
Post operative day & 20.5 & 11 & 0.02 \\
\hline
\end{tabular}

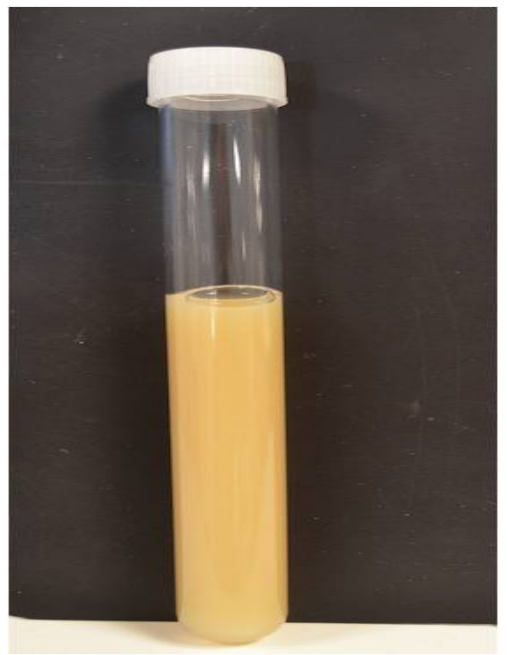

Figure 1. Representative chylous ascites.

chylous ascites. As for the pathological stage, chylous ascites occurred significantly less frequently in the early stage than in the late stage $(\mathrm{p}=0.04)$. There were no significant differences between the groups in operative factors, including operation time and blood loss. As a matter of course, postoperative hospital staywas longer in patients with chylous ascites (20.5 days) than in those without (11 days) $(\mathrm{p}=0.02)$.

\section{Discussion}

The present study showed that chylous ascites after laparoscopic colon surgery required longer hospital stays but could be managed by conservative treatment in all cases.

The main cause of postoperative chylous ascites is considered to be intraoperative injury of the main lymphatic vessels [9]. In general, the incidence rate of chylous ascites after laparoscopic surgery is lower than that previously reported 
after open surgery [2] [9]. The visual magnification provided by the associated equipment makes it easier to identify lymphatic vessels than is possible during open surgery. In addition, many surgeons believe that laparosonic coagulating shears are important for tissue dissection and ligation, including of the lymphatic vessels. The incomplete sealing of the lymphatics with energy devices caused chylous ascites, even though we used by the ultrasonic coagulating shears in our series. In our study, tumor location was not associated with the occurrence of chylous ascites. Some reports, however, found that right hemicolectomy was related to the development of chylous ascites because the lymphatic vessels around the superior mesenteric artery are reportedly abundant [2] [8].

Previous data showed that chylous ascites in patients after colorectal surgery didnot affect their survival [7]. However, a limited report of a small sample size showed a higher rate of cancer recurrence in patients with chylous ascites. Patients who developed chylous ascites after undergoing surgery for pancreatic cancer showed poorer 3-year survival [10].

Chylous ascites prolongs hospital stays and significantly increases treatment costs. Treatment options for chylous ascites are total parenteral nutrition or a medium-chain triglyceride diet with or without octreotide [11]]. We didn't encounter refractory chylous ascites; that is, all of the patients receiving either total parenteral nutrition or the medium-chain triglyceride diet eliminated their chylous ascites. In Japan, octreotide cannot be administered due to health insurance requirements for the improvement of chylous ascites.

Our study has several limitations, including the small number of patients and its retrospective nature. As more data are required to elucidate the mechanism underlying the occurrence of chylous ascites, further studies are warranted.

In conclusion, postoperative hospital stays were longer in patients with chylous ascites than in those without. Late stage of CRC was one of the risk factors for chylous ascites in our study.

\section{Author's Contributions}

Study concept and design (SM); data acquisition (SM, KH, MK, KS, MT, YK, $\mathrm{YT})$; analysis and interpretation of data (SM); drafting of the manuscript (SM); critical revision of the manuscript (KS).

\section{Ethical Approval}

This study was performed in accordance with the ethical standards of the Committee on Human Experimentation of our institution.

\section{Conflicts of Interest}

The authors declare no conflicts of interest regarding the publication of this paper.

\section{References}

[1] Kwaan, M.R., Al-Refaie, W.B., Parsons, H.M., Chow, C.J., Rothenberger, D.A. and 
Habermann, E.B. (2013) Are Right-Sided Colectomy Outcomes Different from Left-Sided Colectomy Outcomes? Study of Patients with Colon Cancer in the ACS NSQIP Database. JAMA Surgery, 148, 504-510. https://doi.org/10.1001/jamasurg.2013.1205

[2] Nishigori, H., Ito, M., Nishizawa, Y., Koyama, A., Koda, T., Nakajima, K., et al. (2012) Postoperative Chylous Ascites after Colorectal Cancer Surgery. Surgery Today, 42, 724-728. https://doi.org/10.1007/s00595-012-0132-x

[3] Matsuda, T., Fujita, H., Kunimoto, Y., Kimura, T. and Ogino, K. (2013) Chylous Ascites as a Complication of Laparoscopic Colorectal Surgery. Asian Journal of Endoscopic Surgery, 6, 279-284. https://doi.org/10.1111/ases.12057

[4] Kaas, R., Rustman, L.D. and Zoetmulder, F.A. (2001) Chylous Ascites after Oncological Abdominal Surgery: Incidence and Treatment. European Journal of Surgical Oncology, 27, 187-189. https://doi.org/10.1053/ejso.2000.1088

[5] Weniger, M., D’Haese, J.G., Angele, M.K., Kleespies, A., Werner, J. and Hartwig, W. (2016) Treatment Options for Chylous Ascites after Major Abdominal Surgery: A Systematic Review. American Journal of Surgery, 211, 206-213.

https://doi.org/10.1016/j.amjsurg.2015.04.012

[6] Yilmaz, M., Akbulut, S., Isik, B., Ara, C., Ozdemir, F., Aydin, C., et al. (2012) Chylous Ascites after Liver Transplantation: Incidence and Risk Factors. Liver Transplantation, 18, 1046-1052. https://doi.org/10.1002/lt.23476

[7] Lee, S.Y., Kim, C.H., Kim, Y.J. and Kim, H.R. (2016) Chylous Ascites after Colorectal Cancer Surgery: Risk Factors and Impact on Short-Term and Long-Term Outcomes. Langenbeck's Archives of Surgery, 401, 1171-1177. https://doi.org/10.1007/s00423-016-1500-6

[8] Baek, S.J., Kim, S.H., Kwak, J.M. and Kim, J. (2013) Incidence and Risk Factors of Chylous Ascites after Colorectal Cancer Surgery. American Journal of Surgery, 206, 555-559. https://doi.org/10.1016/j.amjsurg.2013.01.033

[9] Ablan, C.J., Littooy, F.N. and Freeark, R.J. (1990) Postoperative Chylous Ascites: Diagnosis and Treatment. A Series Report and Literature Review. Archives of Surgery, 125, 270-273. https://doi.org/10.1001/archsurg.1990.01410140148027

[10] Assumpcao, L., Cameron, J.L., Wolfgang, C.L., Edil, B., Choti, M.A., Herman, J.M., et al. (2008) Incidence and Management of Chyle Leaks Following Pancreatic Resection: A High Volume Single-Center Institutional Experience. Journal of Gastrointestinal Surgery, 12, 1915-1923. https://doi.org/10.1007/s11605-008-0619-3

[11] Kuboki, S., Shimizu, H., Yoshidome, H., Ohtsuka, M., Kato, A., Yoshitomi, H., et al. (2013) Chylous Ascites after Hepatopancreatobiliary Surgery. The British Journal of Surgery, 100, 522-527. https://doi.org/10.1002/bjs.9013

\section{Abbreviations}

Colorectal Cancer (CRC). 